\title{
DIGESTION OF NEUTRAL FATS BY HUMAN SUBJECTS ${ }^{1}$
}

\author{
BY ROBERT S. HARRIS, JOHN W. CHAMBERLAIN, AND JAMES H. BENEDICT \\ (From the Food Technology and Medical Departments, Massachusetts Institute of Technology, \\ Cambridge, Mass., and Research Division, Proctor and Gamble Company, \\ Cincinnati, Ohio)
}

(Submitted for publication September 27, 1954; accepted January 12, 1955)

Several theories have been advanced to describe the digestion of fats in animals and in humans. Of these, two have been popular. The hydrolytic theory (1) suggests that triglycerides are broken down completely to fatty acids and glycerol which are then absorbed into the intestinal wall. The particulate theory (2) suggests that triglycerides are partially hydrolyzed in the intestinal lumen to monoglycerides, diglycerides, and fatty acids. The partial glycerides, fatty acids, and bile salts cause a fine emulsification of the remaining triglyceride. This emulsion, containing triglycerides, then enters the intestinal wall.

Much indirect evidence exists for the formation of partial glycerides during fat digestion. Reiser, Bryson, Carr, and Kuiken (3) analyzed the lipids recovered from the thoracic ducts of rats fed tagged triglyceride and concluded that the major portion of ingested fat is hydrolyzed to monoglyceride prior to absorption. Frazer and Sammons (4) noted that lipids isolated from the lumen of the intestinal tract of rats had a high hydroxyl value and concluded that monoglyceride was present. Later Frazer (5) reported, without presenting experimental details, that he had found monoglycerides in the intestinal tracts of human subjects. The formation and presence of monoglycerides in the intestinal tract during the digestion of fat has been reported in the dog (6), rat $(3,4,7-9)$, cat (10), monkey (8), and man $(5,11)$.

Theoretically the formation of two isomeric monoglycerides is possible when a triglyceride is digested to monoglyceride. It was not until the recent work of Mattson, Benedict, Martin, and Beck (7) that 2-monoglycerides were shown to

\footnotetext{
1 Presented in part before the meeting of the American Institute of Nutrition, Federation of American Societies for Experimental Biology, Atlantic City, N. J., April 15, 1954. Contribution No. 256 from the Department of Food Technology, Mass. Institute of Technology, Cambridge, Mass.
}

be present in digestion products. These authors showed that almost all of the monoglycerides formed from 2-oleyl-dipalmitin were initially present as the 2-isomer. This observation indicated that the hydrolysis of triglycerides in the intestinal tract of the rat involved a series of step-wise reactions from triglycerides to 1,2-diglycerides to 2monoglycerides, with fatty acids released at each step. Recently Borgström (12) demonstrated that these same reactions occur during the in vitro hydrolysis of triglycerides by rat pancreatic lipase.

The present study was started in 1951 in order to determine the intermediates formed during fat digestion in humans. It has been found that the level of total monoglyceride ranged up to 13 per cent of the total lipids, and one-half to two-thirds of this was present as 2-monoglyceride. The free fatty acid content was two to three times the total monoglyceride content.

Interfacial tension measurements of the intestinal lipids against the corresponding aqueous phase showed a high level of surface activity during fat digestion.

\section{EXPERIMENTAL}

Healthy, white male subjects, aged 19 to 35 years (mean 24 years), upon entering the Homberg Memorial Infirmary (M.I.T.) early on Friday evening were given morphine sulfate $(1 / 6 \mathrm{gr}$ ) and atropine sulfate $(1 / 150$ gr.) subcutaneously. Their nasal passages were sprayed with a 0.5 per cent solution of Pontocaine and a pledget of cotton moistened with adrenalin inserted. A No. 16 French Miller-Abbott tube, with its rubber balloon containing $3 \mathrm{ml}$. of mercury, was passed nasally. The subjects then ate a supper and went to bed on their right sides. Fluoroscopic examination at about 9 p.m. usually revealed the end of the tube in the first portion of the duodenum. When it was not, the tube was worked into position by manipulating the subject. At 1:00 a.m., $40 \mathrm{ml}$. of air was injected into the balloon to stimulate peristalsis and facilitate passage of the tube along the intestinal tract, and the tube was clamped. This operation was delayed until 1:00 a.m. to allow the evening meal to progress beyond the end of the tube. At this time the subjects usually took $1.5 \mathrm{gr}$. of Nembutal 18 . Pontocaine(8) 
spray was available for use whenever the tube caused distressing irritation of the nose or throat.

On Saturday morning the air was released from the balloon to allow the accumulated intestinal juices to pass. The position of the tube was adjusted under the fluoroscope and an $x$-ray photograph was taken. Later the photograph was used to measure the approximate distance from Treitz' ligament to the most distal hole in the aspirator tube.

The balloon was reinflated and each subject drank 45 ml. of melted vegetable fat ${ }^{2}$ (a 3/1 mixture of soybean oil and cottonseed oil, hydrogenated to an iodine value of 80). The tube was connected to a liter, round-bottom flask containing ethyl ether, and the flask was immersed in an alcohol-dry ice bath. Suction was applied to the system by a Phelan tank operating at a negative pressure of 7 inches of mercury. In preliminary experiments it was established that lipase action ceased completely at this point when only ethyl ether was used; Bloor's mixture of alcohol and ether was ineffective. Periodically (at onehalf or one hour intervals) the collection flasks were exchanged for new ones.

Shortly after each sample was collected more ethyl ether was added to the frozen chyme and the mixture was thawed at $40^{\circ} \mathrm{C}$. When the sample was completely liquefied the bulk of the ether was decanted. Then 3 to 5 volumes of fresh ether and sufficient anhydrous sodium sulfate to absorb all of the water were added to the chyme with vigorous swirling. After filtering, the combined ether extracts were washed five times with water, dried with anhydrous sodium sulfate, filtered and concentrated to approximately $75 \mathrm{ml}$. The samples were placed in 4 oz. bottles with screw caps lined with aluminum foil, packed in dry ice, and shipped to the Procter and Gamble Company laboratories for analysis. There was sufficient dry ice in the package so that some remained at the destination; thus, all samples were kept at a low temperature during transit.

The ether was removed under vacuum at $40^{\circ} \mathrm{C}$. and the residue was weighed. In many instances there was insufficient lipid for analyses. Whenever sufficient material was available a portion of the sample was taken up in chloroform, washed with water, dried with anhydrous sodium sulfate, and analyzed for 1-monoglyceride $(7,13)$. Another portion in chloroform was treated with perchloric acid to convert the 2-monoglycerides to 1 -monoglycerides, then water-washed, and analyzed for total monoglycerides (13). The correction factor to convert from the equilibrium mixture after isomerization to total monoglyceride was not applied in these experiments.

The content of free fatty acids was determined on each fraction by titration.

In all instances whenever samples were not being processed, they were stored at $5^{\circ} \mathrm{C}$.

Measurements of surface activity were made on samples

2 A sample of the test fat was analyzed with each set of intestinal lipid samples. In all cases the free fatty acid content was less than 1.0 per cent and the weight per cent of total and 1-monoglyceride was less than 1.5. obtained as described above except that the chymes were collected in dry flasks immersed in baths of dry ice and alcohol. These chymes, without further processing, were packed in dry ice and shipped to the Procter and Gamble Company laboratories. The frozen samples were allowed to thaw at room temperature and as soon as the samples were pourable they were centrifuged to separate the oily phase from the aqueous phase. The time of centrifugation was less than twenty minutes in all cases. The fractions were immediately stored at $5^{\circ} \mathrm{C}$. until interfacial tension measurements could be made on the lipid against the corresponding aqueous phase. All measurements were made within six hours of centrifugation; it was determined that no change in the values occurred with storage times up to 24 hours. The standard drop volume technique, as described by Andreas, Hauser, and Tucker (14), and Fordham (15), was used for the determination of surface activity in these systems.

\section{RESULTS AND DISCUSSION}

Many preliminary experiments were necessary for the development of satisfactory clinical and laboratory techniques. The following experiments were satisfactory in all respects and were considered to give the most reliable data concerning the intermediates formed during fat digestion in human beings.

The results obtained in eleven experiments with seven male subjects fed melted triglyceride are presented in Table I.

Appreciable quantities of monoglycerides and free fatty acids were present at almost all periods of digestion. The level of total monoglycerides in the intestinal lipids ranged up to 13 per cent with considerable variation among individuals. However, several individuals showed a relatively constant amount, irrespective of the length of time of digestion or the site of chyme recovery. For example, in subject J. E. M. the weight per cent of total monoglyceride ranged from 4.5 to 6.8 whereas in subject W. V. C. it ranged from 10.4 to 11.5 .

The analytical techniques used in these experiments had a limitation which could not be completely overcome. The method for isomerizing 2-monoglycerides to 1-monoglycerides was not quantitative. Under the conditions employed the conversion was generally about 85 per cent complete. Thus, the values for total monoglycerides, as determined by the periodic acid oxidation method, are minimal values.

An appreciable portion of the monoglycerides was of the 2-configuration. In many cases the 
DIGESTION OF NEUTRAL FATS BY HUMAN SUBJECTS

TABLE I

Intestinal lipid analyses at various intervals following the feeding of triglycerides*

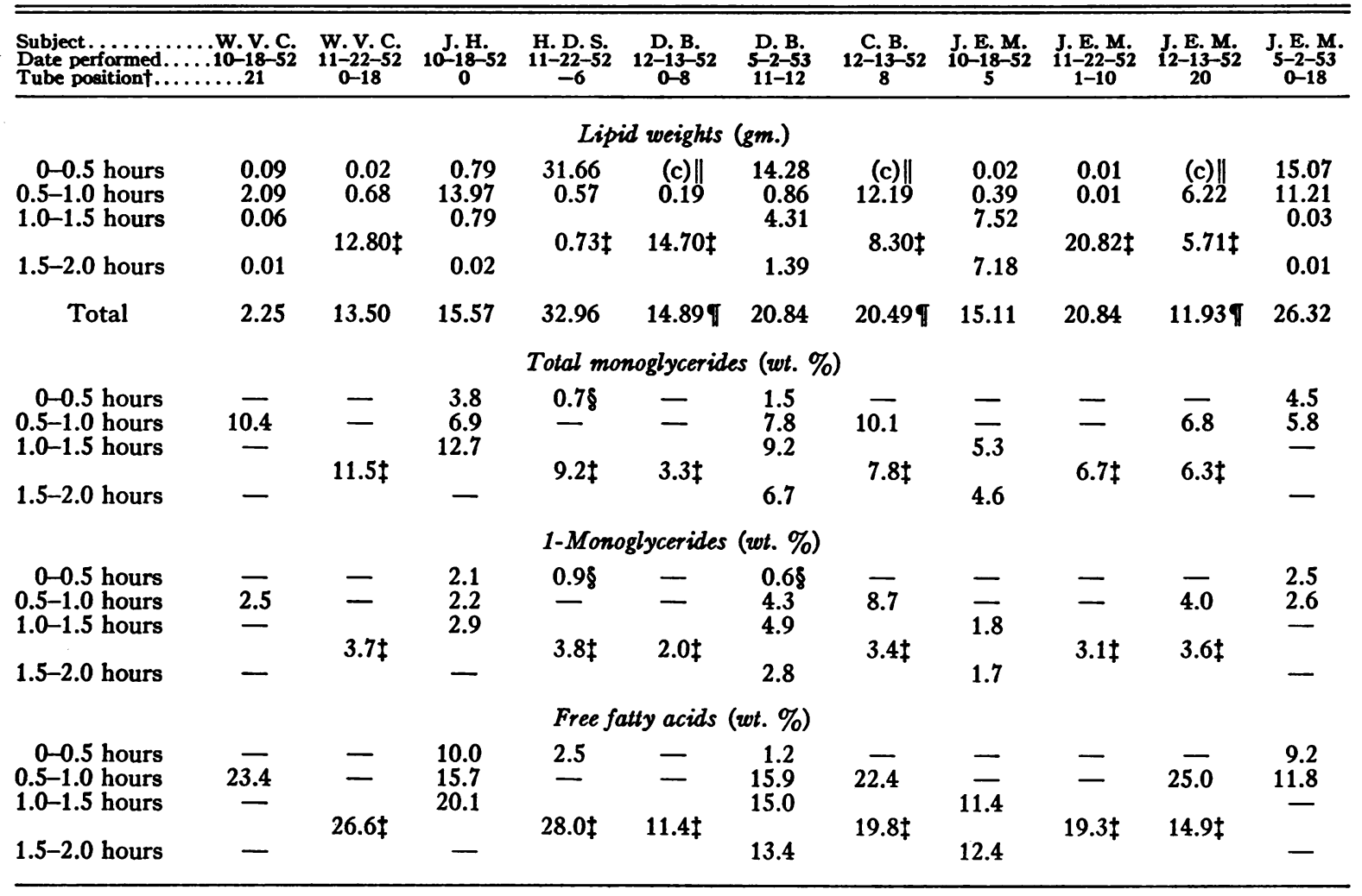

* A 1/3 mixture of cottonseed oil and soybean oil, hydrogenated to an iodine value of 80 .

$\dagger$ Distance in inches between Treitz' ligament and the distal hole of aspirator tube.

$\ddagger$ Samples collected over a one-hour period: 1.0 to 2.0 hours.

These monoglyceride values are below the sensitivity of the method and therefore are not significant.

Lipids were not recovered from this sample.

I Total not complete since initial sample weight was not obtained.

amount of 2-monoglyceride was greater than the amount of 1-monoglyceride. The analytical limitation discussed above makes these minimal values for 2-monoglyceride.

In two experiments (H. D. S. and D. B. 5-2-53) the major portion of the fat was recovered within the first half hour. The very low levels of total monoglyceride and free fatty acids in these samples indicate a limited hydrolysis. This probably resulted from a very rapid passage of fat from the stomach into the intestine with only a limited opportunity for hydrolysis before the fat was recovered.

The level of the free fatty acids was nearly in direct proportion to the amount of total monoglycerides, the ratio of fatty acids to monoglycerides being between $2: 1$ and $3: 1$ for all subjects.

The monoglyceride and free fatty acid levels and types reported here are similar to those ob- served by Mattson, Benedict, Martin, and Beck (7) in rats three hours after feeding triglyceride. Thus, the nature and the levels of these intermediates formed during the intestinal digestion of triglycerides are similar in man and in rats.

It might be expected that the amount of fat recovered from the lower portions of the small intestines would be significantly less than that recovered from the upper end of the intestinal tract, since there would have been more opportunity for fat absorption to occur. Tube positions were compared with fat recoveries but the data were too variable to reveal whether there was a relation between the site of fat recovery and the total amount of fat found.

In the tables where two tube positions are cited, the first value represents the initial position, and the second indicates the location of the tube at the conclusion of the experiment. Where only one 
tube position is given, the value always represents the initial position. Because there was a tendency for the intestine to "sleeve onto" the MillerAbbott tube during the course of each experiment these measurements are necessarily approximate.

In the experiments described above a relatively large amount of fat was fed as the sole component of the test meal. The digestion of fat in a more conventional meal was studied in three subjects who received a breakfast consisting of toast prepared from fat-free bread, $45 \mathrm{~g}$. of the above triglyceride (colored and flavored to resemble margarine), jelly, sugar, and coffee. The collection of the intestinal chymes was started with the initial ingestion of food. Total lipid recoveries (Table II) were considerably lower than when fat alone was fed, but the proportions of monoglycerides and free fatty acids were similar to the previous values. In the one instance (J. E. M.) where a direct comparison was possible, the total monoglyceride and the free fatty acid values were

TABLE II

Intestinal lipid analyses at various intervals following the feeding of a breakfast *

\begin{tabular}{|c|c|c|c|}
\hline $\begin{array}{l}\text { Subject. } \\
\text { Tube positiont.... }\end{array}$ & ${ }_{6-12}^{E} \mathrm{M}$ & $\begin{array}{l}\text { L. G. } \\
\text { 18-24 }\end{array}$ & $\begin{array}{l}\text { R.T. } \\
20-23 \\
\end{array}$ \\
\hline \multicolumn{4}{|c|}{ Lipid weights (gm.) } \\
\hline $\begin{array}{l}0-1.5 \text { hours } \ddagger \\
1.5-2.5 \text { hours } \\
2.5-3.5 \text { hours } \\
3.5-4.2 \text { hours }\end{array}$ & $\begin{array}{l}1.69 \\
1.07 \\
4.98 \\
0.36\end{array}$ & $\begin{array}{l}4.76 \\
0.52 \\
0.31 \\
0.58\end{array}$ & $\begin{array}{l}1.66 \\
1.39 \\
1.38 \\
0.76\end{array}$ \\
\hline Total & 8.10 & 6.17 & 5.18 \\
\hline \multicolumn{4}{|c|}{ Total MG. (wt. \%) } \\
\hline $\begin{array}{l}0-1.5 \text { hours } \\
1.5-2.5 \text { hours } \\
2.5-3.5 \text { hours } \\
3.5-4.2^{2} \text { hours }\end{array}$ & $\begin{array}{r}7.3 \\
9.1 \\
10.2 \\
-\end{array}$ & $\begin{array}{r}5.1 \\
3.1 \\
18.7\end{array}$ & $\begin{array}{r}4.4 \\
11.3 \\
11.2 \\
10.8\end{array}$ \\
\hline \multicolumn{4}{|c|}{$1-M G .(w t . \%)$} \\
\hline $\begin{array}{l}0-1.5 \text { hours } \\
1.5-2.5 \text { hours } \\
2.5-3.5 \text { hours } \\
3.5-4.2 \text { hours }\end{array}$ & $\begin{array}{l}3.9 \\
3.8 \\
5.7 \\
-\end{array}$ & $\begin{array}{l}2.3 \\
1.7 \\
5.6\end{array}$ & $\begin{array}{l}2.4 \\
6.0 \\
6.4 \\
3.5\end{array}$ \\
\hline
\end{tabular}

0-1.5 hours

1.5-2.5 hours

2.5-3.5 hours

$3.5-4.2$ hours

Free fatty acids (wt. \%)

17
20
22
-

10
$\frac{6}{30}$

* Breakfast: toast, 45 gm. "dry" margarine prepared from triglyceride (see text), jelly, coffee, and sugar.

$\dagger$ Inches between Treitz' ligament and distal hole of aspirator of Miller-Abbott tube.

$\ddagger$ Collection of chyme started when subjects began eating breakfast which extended over the first half hour.
TABLE III

Surface actioity at oil-water boundaries in intestinal chymes

\begin{tabular}{lccc}
\hline Subject & $\begin{array}{c}\text { Date } \\
\text { performed }\end{array}$ & $\begin{array}{c}\text { Average time } \\
\text { after fat } \\
\text { ingestion }\end{array}$ & $\begin{array}{c}\text { Interfacial } \\
\text { tension } \\
\text { (dymes cm.-1) }\end{array}$ \\
\hline J.E. M. & $12-13-52$ & 15 min. & 1.3 \\
W. V. C. & $1-17-53$ & 1 hour & $<0.3$ \\
D. B. & $1-17-53$ & 1 hour & $<0.3$ \\
L. G. & $1-17-53$ & 1 hour & $<0.3$ \\
L. G. & $1-17-53$ & 1 hour 45 min. $<0.3$ \\
\hline
\end{tabular}

slightly higher following the feeding of the breakfast than when triglyceride alone was fed. All in all, there was no significant difference in the proportion of fat digestion products found when fat was fed alone and when fat was incorporated into a meal.

Kuhrt and his co-workers (11) have reported the presence of 38 per cent and 50 per cent monoglyceride in the intestinal contents of two subjects after eating a light lunch. Apparently 2-monoglyceride was absent, for the total monoglyceride appeared to be all 1-monoglyceride. The striking differences in monoglycerides values between the observations of Kuhrt and his associates and this study may be attributed to the distinct differences in the nature of the test meal, and in the methods of chyme collection, lipid isolation and lipid analysis.

The interfacial tension values of five samples obtained from subjects fed a melted triglyceride are given in Table III. The values reported are for unaged surfaces. If diffusion between surfaces had been allowed to take place until equilibrium was attained, still lower interfacial tensions would have been observed $(14,15)$. The data show that the level of surface activity during fat digestion was high. The previous work of Dasher (16) indicates that the bile salts are largely responsible for this high surface activity. In fact, the surface activity of the intestinal contents during the digestion of fat is so high that large quantities of monoglyceride of dietary origin could be present without significantly lowering the boundary tensions.

\section{SUMMARY}

Lipids recovered from the lumen of the intestines of eleven healthy young men were analyzed for content of free fatty acids, total monoglycerides, and 1-monoglycerides. 
The level of total monoglyceride ranged up to 13 per cent of the total lipids, and one-half to two-thirds of this was present as 2-monoglyceride. The free fatty acid content was two to three times the total monoglyceride content.

Three subjects were fed a breakfast of toast, margarine, jelly, sugar, and coffee. There was no significant difference in the proportion of fat digestion products recovered when fat was fed alone and when incorporated into a meal.

Interfacial tension measurements made on intestinal lipids against the corresponding aqueous phases showed a high level of surface activity during fat digestion.

\section{ACKNOWLEDGMENTS}

The authors wish to thank Dr. G. F. Dasher for obtaining the surface activity data reported herein.

The participation of the many who volunteered as subjects for this research and submitted to discomfort without complaint is especially appreciated by the investigators.

\section{REFERENCES}

1. Verzár, F., and McDougall, E. J., Absorption from the Intestine. London, Longmans, Green, and Co., 1936.

2. Frazer, A. C., Schulman, J. H., and Stewart, H. C., Emulsification of fat in the intestine of the rat and its relationship to absorption. J. Physiol., 1944, 103, 306.

3. Reiser, R., Bryson, M. J., Carr, M. J., and Kuiken, $\mathrm{K}$. A., The intestinal absorption of triglycerides. J. Biol. Chem., 1952, 194, 131.

4. Frazer, A. C., and Sammons, H. G., The formation of mono- and di-glycerides during the hydrolysis of triglycerides by pancreatic lipase. Biochem. J., 1945, 39, 122.

5. Frazer, A. C., Aetiology of steatorrhea. Brit. M. J., 1947, 2, 641.

6. Desnuelle, P., Hydrolyse enzymatique des triglycérides. Bull. Soc. chim. biol., 1951, 33, 909.

7. Mattson, F. H., Benedict, J. H., Martin, J. B., and Beck, L. W., Intermediates formed during the digestion of triglycerides. J. Nutrition, 1952, 48, 335.

8. Favarger, P., Collet, R. A., and Cherbuliez, E., Etude de la résorption intestinale des graisses à l'aide de deutéro-glycérol et d'acides gras marqués. Helvet. chim. acta, 1951, 34, 1641.

9. Borgström, B., On the action of pancreatic lipase on triglycerides in vivo and in vitro. Acta physiol. Scandinav., 1952, 25, 328.

10. Bergström, S., Borgström, B., Carlsten, A., and Rottenberg, M., On the mechanism of the intestinal fat absorption. Acta chem. Scandinav., 1950, 4, 1142.

11. Kuhrt, N. H., Welch, E. A., Blum, W. P., Perry, E. S., Weber, W. H., and Nasset, E. S., Isolation and identification of monoglycerides in the intestinal contents of humans. J. Am. Oil Chem. Soc., 1952, 29, 271.

12. Borgström, B., On the mechanism of the hydrolysis of glycerides by pancreatic lipase. Acta chem. Scandinav., 1953, 7, 557.

13. Martin, J. B., The equilibrium between symmetrical and unsymmetrical monoglycerides and determination of total monoglycerides. J. Am. Chem. Soc., 1953, 75, 5483.

14. Andreas, J. M., Hauser, E. A., and Tucker, W. B., Boundary tension by pendant drops. J. Phys. Chem., 1938, 42, 1001.

15. Fordham, S., The calculation of surface tension from measurements of pendant drops. Proc. Roy. Soc., London, 1948, A194, 1.

16. Dasher, G. F., Surface activity of naturally occurring emulsifiers. Science, 1952, 116, 660. 